be generally true and could depend on the magnitude of the liquid-liquid interactions. In the light of these observations it was decided not to extend the theory at this time to encompass ternary systems as such.

Further work is in progress to establish the validity of the various points raised in this study.

Acknowledgment. The authors thank the S. R. C. for financial support during this work, in the form of a fellowship to I. J. M.

\section{References and Notes}

(1) J. M. G. Cowie and J. T. McCrindle, Eur. Polym. J., 8, 1185 (1972)

(2) J. M. G. Cowie and I. J. McEwen, J. Chem. Soc., Faraday Trans 1, 70, $171(1974)$.
(3) J. M. G. Cowie and J. T: McCrindle, Eur. Polym. J., 8, 1325 (1972).

(4) A. Dondos and D. Patterson, J. Polym. Sci., Part A2, 7, 209 (1969).

(5) D. Patterson and G. Delmas, Trans. Faraday Soc., 65, 708 (1969); D. Patterson, J. Polym. Sci., Part C, 16, 3379 (1968)

(6) P. J. Flory, Discuss. Faraday Soc., 49, 7 (1970).

(7) J. M. G. Cowie, Pure Appl. Chem., 23, 355 (1970)

(8) Z. Tuzar and M. Bohdanecky, Collect Czech. Chem. Commun., 34, 289 (1969).

(9) A. R. Shultz and P. J. Flory, J. Amer. Chem. Soc, 74, 4760 (1952).

(10) K. S. Siow, G. Delmas, and D. Patterson, Macromolecules, 5, 29 (1972).

(11) N. Kuwahara, S. Saeki, S. Konno, and M. Kaneko, Macromolecules, 6, 246 (1973).

(12) C. M. Hansen, Chem. Technol., 2, 547 (1972)

(13) A. Zivny, J. Pouchly, and K. Solc, Collect. Czech. Chem. Commun., 32, 2753 (1967)

(14) P. J. Flory and H. Hocker, Trans. Faraday Soc., 67, 2258 (1971).

(15) H. Hocker and P. J. Flory, Trans. Faraday Soc., 67, 2270 (1971)

\title{
Computer Graphics in the Solution of the Chain Deformation Problem
}

\author{
Steven D. Stellman \\ Department of Biochemical Sciences, Frick Chemical Laboratory, Princeton University, \\ Princeton, New Jersey 08540. Received July 2, 1973
}

\begin{abstract}
Gō and Scheraga have given a general solution for determining the possible conformations of a section of a flexible polymeric chain between two fixed bonds (if the bonds coincide, the problem is equivalent to ring closure). Often, the fixed bonds are related by symmetry, such as that imposed by helicity or folding of antiparallel chains. A technique is shown for obtaining solutions using interactive computer graphics, whereby a plausible chain conformation or fold is approximated manually and completed by mathematical minimization. The procedure allows rapid, repetitive exploration of multiple solutions, and has many options for changing con. straints. As an example, the backbone conformation of $\mathrm{GpC}$, a dinucleoside phosphate, is used as a starting model, and gives upon minimization a set of backbone angles in the range of 11-fold helical RNA.
\end{abstract}

The chain deformation problem and the mathematically equivalent ring closure problem are of great importance in studies of biopolymers. ${ }^{1}$ The problem arises whenever the geometrical relationship of two monomeric units to each other is known, for example, through a postulated hydrogen-bonding scheme, or by symmetry, and it is required to find the coordinates of atoms along or connected to the backbone chain between the fixed units.

A simple method of solution would obviously be of benefit in building models for chain folding of $\mathrm{tRNA}^{2}$ or proteins, ${ }^{3}$ and indeed any case where the groups (nucleotide bases or amino acids) on either side of the fold region are fixed with respect to each other.

In this paper we demonstrate a simple yet effective method for determining possible conformations of backbone atoms between fixed base units of a helical polynucleotide chain. The method is perfectly general and could be applied to any other polymeric system, such as a protein. While we have exploited the method specifically in our model building work with computer graphics, the mathematics are perfectly adaptable to nongraphical systems. However, computer graphics has unique advantages in the applications for which our model-building programs were developed.

\section{Conformations of Polynucleotides}

The backbone chain of a helical polynucleotide such as DNA contains many bonds about which semihindered or free rotation can occur. Consequently, a large (perhaps infinite) number of conformations exists which satisfy reasonable stereochemistry.

If the helix pitch and unit cell length in the fiber axis direction are determined for fibers of this polymer through $\mathrm{X}$-ray fiber diffraction, then the cylindrical coordinates of the base and backbone atoms are usually obtained by manually building wire models consistent with these data, ruling out those models which are stereochemically impossible, and refining the remaining models through a least-squares procedure. ${ }^{4,5}$

The deformation problem enters the situation because, given the absolute orientation of, say, one base with respect to the fiber axis, the coordinates of the next and every succeeding base are uniquely fixed by helical symmetry. The problem is then to find possible conformations of the sugar-phosphate backbone which fit in between two adjacent bases and which are stereochemically acceptable (in terms of near-standard bond lengths and bond angles).

Digital computers have long been used to generate the accurate coordinates of such models from initial data measured on stiff-wire models, and to refine the models against observed X-ray diffraction data. ${ }^{6}$ Recently, computers employing interactive three-dimensional graphics have been used to generate complete models themselves. ${ }^{7}$ An obvious advantage of this procedure is to circumvent the hazards of real models, such as gravitational collapse, inflexible bond lengths and bond angles, and poorly measurable dihedral angles. Fast calculation of interatomic overlaps is also possible. ${ }^{8}$

In the following section we describe a method for constructing such models directly comparable with X-ray diffraction data. Given the screw and fiber axis length as initial conditions, and allowing the orientation of the base with respect to the fiber axis and its distance therefrom as adjustable parameters, the deformation problem is solved 
to give the allowable conformations of DNA or RNA sugar-phosphate backbone.

\section{Mathematical Statement of the Problem}

A typical base-ribose-phosphate unit is shown in Figure 1 , with the definition of dihedral angles. We define the helix parameter $\Delta Z$ as the fiber repeat distance ( $z$ axis taken to be the fiber axis or $c$ axis), and $\Delta \Phi$ equal to $360^{\circ} / p$, where $p$ is the unit repeat or "foldness" of the helix (e.g., $p=12$ for a 12 -fold helix, $\Delta \Phi_{12}=360^{\circ} / 12=$ $30^{\circ}$ ). Assuming all bases are planar, we use the notation of Arnot $t^{5}$ for defining the orientation of the base plane by three angles: $\theta_{1}$ (tilt), $\theta_{2}$ (twist), and $\theta_{3}$ (swivel). The distance $D$ of the base from the fiber axis is the fourth required parameter.

When the coordinates of any nucleotide are given, then the cylindrical coordinates of every atom of the next nucleotide are uniquely determined from the corresponding atoms in the first by the relations

$$
\begin{gathered}
r_{2}=r_{1} \\
\phi_{2}=\phi_{1}+\Delta \Phi \\
z_{2}=z_{1}+\Delta Z
\end{gathered}
$$

Counting the $\mathrm{C}_{3}{ }^{\prime}-\mathrm{C}_{4}{ }^{\prime}$ bond as the first, there are six consecutive bonds about which more or less free dihedral rotation may occur. The glycosidic angle connecting sugar with base and the puckering of the sugar are additional variables. However, the values which all those angles may simultaneously assume is severely limited by the backbone's connectivity. In practice, there is usually only a small finite number of possible conformations of the backbone which satisfy stereochemistry, and yet preserve connectivity.

Gō and Scheraga determined these solutions in the following manner. They placed a local cartesian coordinate system on each backbone-chain atom, and considered the possibility of changing the $n$ consecutive dihedral angles $\omega_{1}, \cdots, \omega_{n}$ located in the middle of a long-chain molecule without disturbing the locations and orientations of the local coordinate systems other than those numbered 1 to $n$ - 1. In particular, the locations and orientations of the local coordinate systems numbered 0 and $n$ should remain fixed for any deformations of the intervening atoms.

Let $\mathbf{s}_{n}$ be the position vector of the $n$th coordinate system, $\mathbf{u}_{n}$ be the unit vector in the $x$ direction for that system (taken to be along the bond connecting atom $n-1$ with $n)$, and $\mathbf{v}_{n}$ the unit vector in the local $y$ direction. The set of vectors $\left\{\mathbf{s}_{n}, \mathbf{u}_{n}, \mathbf{v}_{n}\right\}$ exactly specifies the position and orientation of the $n$th local coordinate system. This vector set contains only six degrees of freedom, because of the internal relations

$$
\begin{gathered}
\mathbf{u} \cdot \mathbf{u}=\mathbf{v} \cdot \mathbf{v}=1 \\
\mathbf{u} \cdot \mathbf{v}=0
\end{gathered}
$$

Since coordinate systems 0 and $n$ are fixed, and assumed known, the location and orientation of coordinate system $n$ relative to coordinate system 0 is given by

$$
\begin{aligned}
\mathbf{s}_{n} & =\mathbf{p}=\text { const } \\
\mathbf{u}_{n} & =\mathrm{T} \mathbf{e}_{1}=\text { const } \\
\mathbf{v}_{n} & =\mathrm{Te}_{2}=\text { const }
\end{aligned}
$$

where $\mathbf{p}$ is a constant vector and $\mathbf{T}$ a constant orthogonal transformation matrix. $\mathbf{e}_{1}$ and $\mathbf{e}_{2}$ are column vectors whose transposes are given by $\mathbf{e}_{1}^{\mathrm{T}}=(1,0,0), \mathbf{e}_{2}^{\mathrm{T}}=(0,1,0)$.

The connectivity of the chain itself acts as a constraint on eq 2 , so that the vector connecting atoms 0 and $n$ can be written as the surn of all the intermediate bond vectors. If each of these bond vectors is referred to the frame

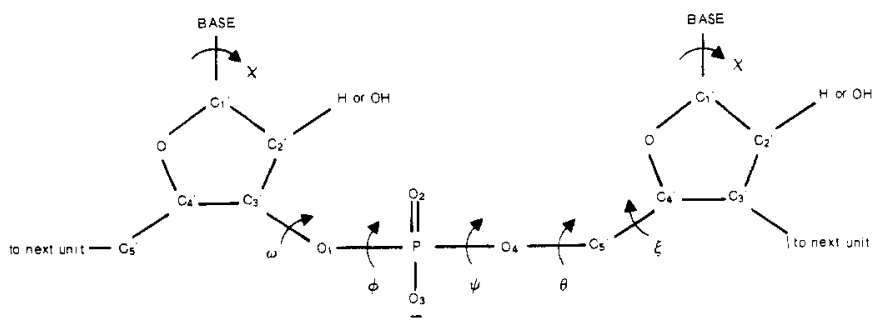

Figure 1. Stereochemically independent backbone unit of DNA or RNA, showing dihedral angles. Angle naming convention is that of Arnott. ${ }^{5}$

of reference of atom 0 , then the three vectors $\mathbf{s}_{n}, \mathbf{u}_{n}$, and $\mathbf{v}_{n}$ can be written as an iterated matrix expression containing the individual matrices and vectors for successive transformation by orthogonal rotation and translation all along the chain. In other words

$$
\begin{aligned}
\mathbf{s}_{n}=\mathbf{p}_{0}+\mathbf{T}_{0} \mathbf{R}_{1} \mathbf{p}_{1} & +\mathbf{T}_{0} \mathbf{R}_{1} \mathbf{T}_{1} \mathbf{R}_{2} \mathbf{p}_{2}+\cdots+\mathbf{T}_{n-2} \mathbf{R}_{n-1} \mathbf{p}_{n-1} \\
\mathbf{u}_{n} & =\mathbf{T}_{0}\left[\prod_{k=1}^{n-1} \mathbf{R}_{k} \mathbf{T}_{k}\right] \mathbf{R}_{n} \mathbf{e}_{1} \\
\mathbf{v}_{n} & =\mathbf{T}_{0}\left[\prod_{k=1}^{n-1} \mathbf{R}_{k} \mathbf{T}_{k}\right] \mathbf{R}_{n} \mathbf{e}_{2}
\end{aligned}
$$

The matrices $\mathbf{T}$ and $\mathbf{R}$ are given by

$$
\begin{aligned}
\mathbf{T}_{i} & =\left[\begin{array}{lll}
\cos \theta_{i} & -\sin \theta_{i} & 0 \\
\sin \theta_{i} & \cos \theta_{i} & 0 \\
0 & 0 & 1
\end{array}\right] \\
\mathbf{R}_{i} & =\left[\begin{array}{lll}
1 & 0 & 0 \\
0 & \cos \omega_{i} & -\sin \omega_{i} \\
0 & \sin \omega_{i} & \cos \omega_{i}
\end{array}\right]
\end{aligned}
$$

The translation vector $\mathbf{p}$ is given by

$$
\mathbf{p}_{i}=\left[\begin{array}{l}
d_{i} \\
0 \\
0
\end{array}\right]
$$

In the above expressions, $d_{i}, \theta_{i}$, and $\omega_{i}$ are the bond distance, angle, and dihedral angle relating the $(i-1)$ coordinate system to the $i$ th. Equating quantities in (2) and (3) gives a system of nine equations, containing three dependent relationships among the center dot products, or six independent equations altogether. Therefore, the number of independent unknowns is $n-6$.

\section{Gō and Scheraga Solution}

The procedure adopted by Gó and Scheraga for the solution of eq 3 is first to select arbitrarily $n-6$ of the $n$ dihedral angles as the independent variables. An arbitrary set of values is then assigned to those variables, and the six independent equations are solved for the remaining six dihedral angles.

Because of the transcendental nature of the equations, there are often multiple solutions which satisfy the original constraints. It occasionally happens that no solutions exist. Conditions for the existence of solutions are stated in ref 1 as a set of rather complicated algebraic inequalities; it may take nearly as much computation to determine the existence of solutions as to obtain the solutions themselves. Actual solution of the equations is performed by first blocking out eq 3 so that four of the equations contain only four variables, viz., $\omega_{1}$ to $\omega_{4}$. Then $\omega_{2}, \omega_{3}$, and $\omega_{4}$ are each expressed in terms of $\omega_{1}$, and the equation for $\mathbf{s}$ is written as a function of $\omega_{1}$ only. Provided so- 
Table I

Results of Minimization of Two Trial Conformations for RNA

\begin{tabular}{|c|c|c|c|c|c|c|c|}
\hline & & $D=4$ & $\begin{array}{c}\Delta Z=3 \\
\text { Base or } \\
\theta_{1}=13 \\
\text { iral Angl }\end{array}$ & $\begin{array}{l}\text { rameters } \\
\Delta \Phi=3 \\
\text { on paran } \\
\theta_{2}=-4 \\
\text { ) }\end{array}$ & $\theta_{3}=-74.5^{\circ}$ & & \\
\hline & $x$ & $\xi$ & $\theta$ & $\psi$ & $\phi$ & $H$ & Ref \\
\hline $\begin{array}{l}\text { Initial } \\
\text { Final }\end{array}$ & $\begin{array}{l}74.76 \\
93.31\end{array}$ & $\begin{array}{r}49.46 \\
5.03\end{array}$ & $\begin{array}{r}\text { Tri } \\
186.47 \\
69.57\end{array}$ & $\begin{array}{r}293.97 \\
75.11\end{array}$ & $\begin{array}{l}292.96 \\
248.68\end{array}$ & $\begin{array}{l}9.81 \\
2 \times 10^{-7}\end{array}$ & \\
\hline $\begin{array}{l}\text { GpC (mol 1) } \\
\text { Initial }^{a} \\
\text { Final } \\
\text { (11-fold helical } \\
\text { RNA) }\end{array}$ & $\begin{array}{l}74.9 \\
73.48 \\
73.50 \\
74.9\end{array}$ & $\begin{array}{l}44.9 \\
50.27 \\
53.11 \\
48.1\end{array}$ & $\begin{array}{l}\quad \text { Tri } \\
175.1 \\
173.32 \\
185.21 \\
179.8\end{array}$ & $\begin{array}{l}293.6 \\
281.60 \\
292.88 \\
297.9\end{array}$ & $\begin{array}{l}287.4 \\
271.76 \\
289.91 \\
285.7\end{array}$ & $1.1 \times 10^{-3}$ & 12 \\
\hline
\end{tabular}

a Set as close as possible to $\mathrm{GpC}$, within error of potentiometer knobs.

lution(s) exist for $\omega_{1}$, the remaining unknowns are obtained by substituting solved values of $\omega_{1}$ in the respective equations.

\section{Solution by Computer Graphics}

Despite the obvious importance of the Gō-Scheraga solution as an advance in model building and geometric construction techniques for macromolecules, it has several disadvantages which limit its general usefulness. (1) There is no systematic search procedure for multiple solutions. For example, for a polypeptide, there are at least two independent angles which must be chosen arbitrarily and given arbitrary values. "Best" choices of those angles and values can be a matter of luck. (2) Analytical indication that no solution exists for given values of the independent $\omega$ 's cannot reveal the possibility that acceptable solutions exist nearby. (3) Relaxation of assumed constraints such as fixed bond angles is not a part of their scheme, although it could obviously be programmed. In this case, however, we mean changes of constraints suggested by closeness to a solution. Here, visualization of a model is critical, since the number of possible parameters which could be allowed to vary is quite large.

In this section we present a new approach to modeling of helical macromolecules which is mathematically equivalent to the Gō-Scheraga method for dealing with chain deformation. The method has proved useful in the trialand-error method of Fourier structure analysis ${ }^{5}$ based on models constructed by computer graphics.

We have previously described a computer system for interactive three-dimensional computer graphics in molecular biology. ${ }^{7}$ Briefly stated, computer graphics is a system for displaying programmed coordinates and lines in perspective on a television-type screen. Use of specialized input devices such as knobs and switches in a time-shared environment provides a continuous means of updating the computer memory with fresh input, such as bond angles, or logical values to control the course of program execution.

The model-building program, called HLX, allows the user to specify the $c$-axis repeat and number of nucleotides per repeat $(\Delta Z$ and $\Delta \Phi)$. The orientation of the base (any of $A, G, C$, or $U$-the choice does not matter) is defined by tilt, twist, swivel, and distance from the helix axis, which may be set by the user. Ribose puckering may be selected from among 40 different conformations, spanning a complete pseudorotation coordinate. ${ }^{9}$

The viewer now observes two nucleotide units on the screen, one displaced from the other by the specified screw-axis symmetry. A photograph of the screen at this stage is displayed in Figure 2a. The user's task is to connect the sugar-phosphate-sugar backbone connecting the two bases by joining the loose end of the first base to the appropriate atom of the second. The individual bonds comprising the backbone chain may be manipulated by potentiometer knobs acting as input devices. Each knob can be rotated a full $360^{\circ}$, and the value of the knob at a given instant is read by the computer and translated into a dihedral angle rotation on the screen. Angles controlled by different knobs are $\chi, \xi, \theta, \psi$, and $\phi$ (see Figure 1 for definitions). Because of eq 1 , the angle $\omega$ depends implicitly on the others and is not needed for input. Values of the current orientation parameters are displayed as text.

The actual coordinates of the sugar and phosphate atoms are calculated from the input dihedral angles using the iterated transform method of Scott and Scheraga, ${ }^{10}$ with fixed bond lengths and bond angles adopted from known geometries of small molecules, or from previously refined polynucleotide data.

The $\mathrm{O}_{1}-\mathrm{C}_{3}{ }^{\prime}$ bond of the second nucleotide is brightened on the screen and acts as a target for the last bond of the adenosine phosphate tail. The degree of coincidence of these two bonds is calculated by a "helix criterion function"

$$
H=\sum_{j=1}^{3}\left(\mathrm{O}_{1 j}{ }^{(1)}-\mathrm{O}_{1 j}{ }^{(2)}\right)^{2}+\sum_{j=1}^{3}\left(\mathrm{C}_{3 j}{ }^{(1)}-\mathrm{C}_{3 j}{ }^{(2)}\right)^{2}
$$

where $j$ runs over $x, y$, and $z$ coordinates, the superscript (1) applies to the atom at the tail of the first nucleotide, and (2) refers to the same atom at the beginning of the second nucleotide.

$H$ is a nonnegative function of all the independent variables (dihedral angles). When $H$ is exactly zero, the two bonds (calculated and target) exactly coincide, and the helical constraints are thereby satsified. Any set of angles for which this is true is thus a solution of the Gō-Scheraga problem. That the two methods are mathematically equivalent is seen by the fact that $\mathrm{H}$ depends on six independent coordinates, viz., the three cartesian coordinates for $\mathrm{O}_{1}$ and the three for $\mathrm{C}_{3}{ }^{\prime}$. Specification of these six variables, which depend implicitly on all the intervening dihedral angles in between the two bases, requires the same amount of information implied in eq 2, and in fact could be used to calculate $\mathbf{s}, \mathbf{u}$, and $\mathbf{v}$.

The problem is slightly complicated by the fact that the knob reading for $\chi$ must also be transmitted to the second displayed nucleotide. The brightened bond can thus change position whenever $\chi$ is changed, and becomes a moving target.

Once the user has come close to a solution, as evidenced 

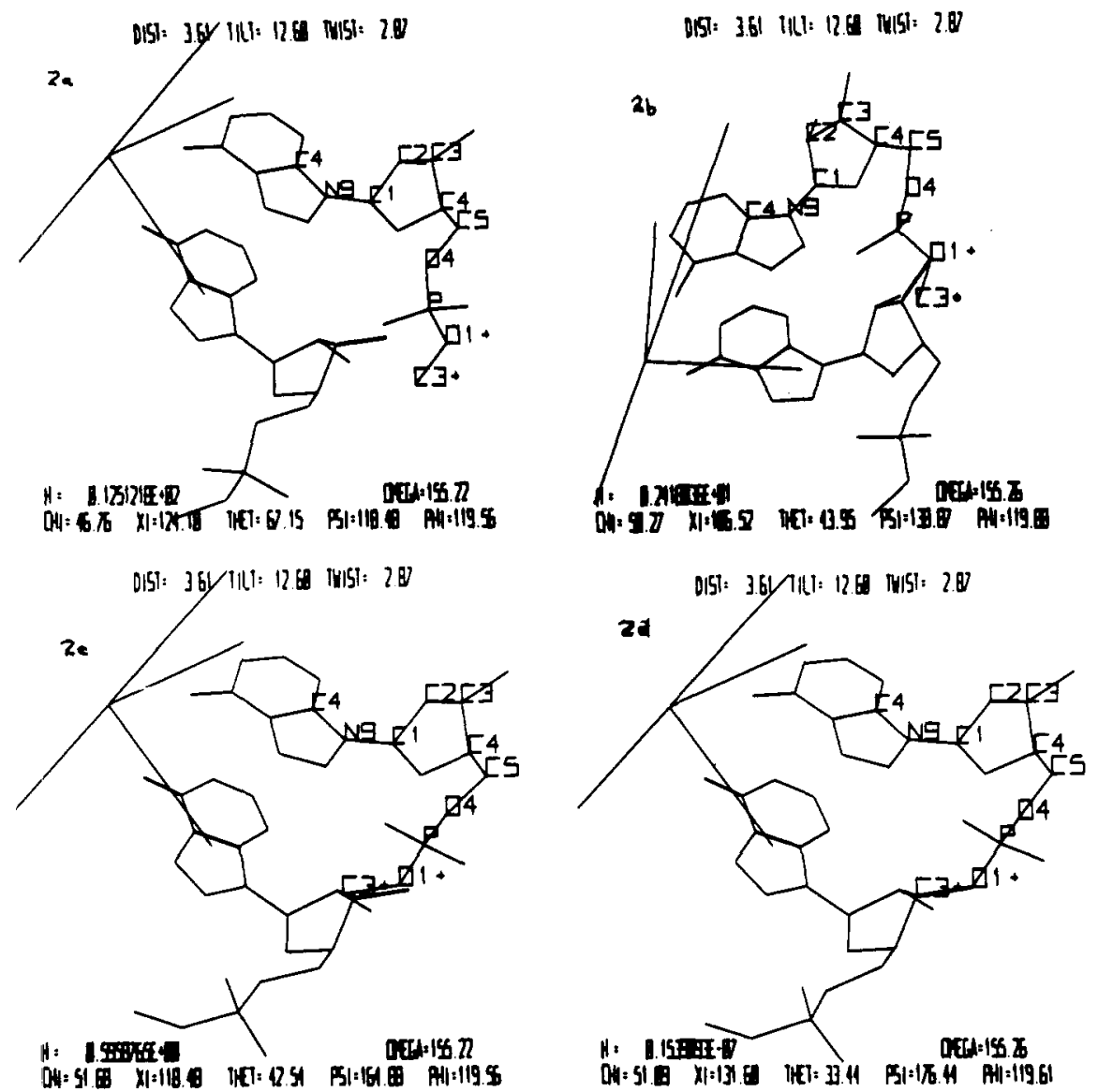

Figure 2. Example of interactive computer graphics solution of polynucleotide conformation. Programmer specifies helix parameters $\Delta Z$ and $\Delta \Phi$, and orientation parameters $D, \theta_{1}, \theta_{2}$, and $\theta_{3}$ (distance from helix axis, tilt, twist, and swivel). (a-c) Successive stages of manual adjustment of backbone dihedral angles. The object is to make the trailing bond $\mathrm{O}_{1}+-\mathrm{C}_{3}+$ coincide with the intensified target bond on the next nucleotide unit, as calculated from the first unit by eq 1. (d) Results of numerical minimization of $H$ via eq 4 , using values displayed in (c) as input.

visually (see Figure 2 for an example), and by a low value of $H$; connectivity is completed by numerical minimization of $H$. The most recent manually set values of the dihedral angles serve as the initial conformation for this routine. The subroutine itself is the Courant-AEC version of Powell's method for minimizing a function of several variables without calculating derivatives. ${ }^{11}$ Typical values of $H$ after minimization are of the order $10^{-8}$. Dividing by four (one less than five dihedral angles) and taking the square root, we have a typical standard deviation in the result of $5 \times 10^{-5} \AA$, a quantity only slightly greater than the machine accuracy.

\section{Further Options}

The above description encompasses the essential features of the method. In applying it to polynucleotides, we have found the following options to be useful. (1) The number of angles varied in minimization of $H$ may be chosen prior to minimization. For example, the user may decide to fix $x$ in the anti conformation. (2) The puckering of the sugar may be changed at any time. (3) The base orientation (tilt, twist, swivel, and $D$ ) may be changed simultaneously with minimization. Thus, the range of orientation parameters which are consistent with chain connectivity may be explored. (4) The three adjacent bond angles about the phosphorus atom can also be varied. This is helpful after a structure has already been minimized once, and typically decreases $H$ by a factor of $10-$ 100 , while relaxing the phosphodiester bond angles by no more than $2-3^{\circ}$.

\section{Example: A Model for 11-fold Helical RNA}

As Gō and Scheraga pointed out, several different sets of dihedral angles satisfying the chain connectivity constraint may be possible, corresponding to multiple solutions of the matrix equations for the positions of the chain backbone atoms. Many of these can often be eliminated on the basis of atomic overlaps observed on the display, or calculated energetically. Each remaining solution must then be considered a possibility and tested against experi- mental data, such as X-ray fiber diffraction data, to determine whether it is the correct one. Furthermore, many different starting conformations such as the one displayed in Figure 2a must be selected and minimized, to assure that most possibilities have been found. The interactive graphics makes this particularly convenient, for the minimization time rarely exceeds $15 \mathrm{sec}$.

As an example, Table I shows the results of two trials in which $\Delta Z$ was set to $30 \AA$ for an 11 -fold helix, with the base orientation parameters set close to those reported ${ }^{6}$ for helical RNA. In the second trial the initial conformational angles were set as close as possible to those reported by Stellman et al. ${ }^{12}$ for the crystal structure of the dinucleoside phosphate, $\mathrm{GpC}$. The minimization results for this trial compare favorably with the most recent set of angles reported for A-RNA by Arnott, Hukins, and Dover. ${ }^{13}$

\section{Discussion of Technique}

The use of these programs has opened up an entirely new approach to helix model building for comparison with 
experiment. Besides the substantial reduction in computational time, we have found that the ability to display and calculate properties of models almost instantly has given us insights into the geometric and energetic properties of the molecules, and enables us to survey and compare more quickly the possible solutions and choose the most likely ones for further study. There are many intramolecular relationships which are immediately obvious when visually displayed but which are obscure or invisible when expressed on paper as numerical coordinates. ${ }^{14}$

The most pressing application is in solution of the structures of crystalline polynucleotides, such as tRNA. Yeast tRNA ${ }^{\text {Phe }}$ has been shown to crystallize in a conformation containing two helical regions orientated at right angles to each other. The solution reported by Rich et al. ${ }^{2}$ is at $3-\AA$ resolution, which does not enable precise definition of atomic coordinates. Further refinement against higher angle X-ray data, essential for comparison with monomer or dimer structures, requires a very good atomic model for a trial structure. The graphical method presented here would be ideal for developing such models, using the helicity parameters and phosphorus atom positions in the helical regions of the $3-\AA$ structure as constraints. This possibility is now being explored with crystallographic data for tRNA available in our laboratory.

Acknowledgments. I thank Dr. Suse Broyde for encouragement and advice. Mr. Edwin Heilweil performed the photographic work. The molecular modeling was performed on the PDP-10/LDS-1 system at the Princeton University Computer Graphics Laboratory, supported by NIH Grant RR-00578. Other support was received from NIH GM-16539 and NSF GB-28021.

\section{References and Notes}

(1) N. Gō and H. A. Scheraga, Macromolecules, 3, 178 (1970).

(2) S. H. Kim, G. J. Quigley, F. L. Suddath, A. McPherson, D. Sneden, J. J. Kim, J. Weinzierl, and A. Rich, Science, 179, 285 (1973).

(3) B. W. Matthews, Macromolecules, 5, 818 (1972); G. Némethy and M P. Printz, ibid., 5, 755 (1972).

(4) R. Langridge, H. R. Wilson, C. W. Hooper, M. H. F. Wilkins, and L. D. Hamilton, J. Mol. Biol., 2, 19 (1960); W. Fuller, M. H. F., Wilkins, H. R. Wilson, and L. D. Hamilton, ibid, 12, 60 (1965)

(5) S. Arnott, Progr. Biophys. Mol. Biol., 21, 265 (1970).

(6) S. Arnott, S. D. Dover, and A. J. Wonacott, Acta Crystallogr., Sect. $B, 25,2192(1969)$

(7) S. D. Stellman, A. M. Lesk, P. J. Bond, S. Harbison, and R. Lan gridge, Proceedings of the First Computer Science Conference, Co lumbus, Ohio, Feb, 1973.

(8) S. D. Stellman, M. Froimowitz, and P. J. Gans, J. Comput. Phys., 7, $178(1970)$.

(9) C. Altona and M. Sundaralingam, J. Amer. Chem. Soc., 94, 8205 (1972).

(10) R. Scott and H. A. Scheraga, J. Chem. Phys, 44, 3054 (1966).

(11) M. Powell, Comput. J., 7, 155 (1964).

(12) S. D. Stellman, B. Hingerty, S. B. Broyde, E. Subramanian, T. Sato, and R. Langridge, Biopolymers, 12, 2731 (1973).

(13) S. Arnott, D. W. L. Hukins, and S. D. Dover, Biochem. Biophys, Res. Commun., 48, 1392 (1972).

(14) P. J. Bond, R. Langridge, A. Lesk, S. Stellman, and S. Harbison, Comput. Graphics, 6 (4), 13 (1972).

\title{
Singularities of the Kirkwood-Riseman Equations of Polymer Hydrodynamics
}

\author{
Robert Ullman \\ Scientific Research Staff, Ford Motor Company, Dearborn, Michigan 48121. \\ Received January 9, 1974
}

\begin{abstract}
The equations of polymer hydrodynamics formulated by Kirkwood and Riseman utilize a collection of point forces which act as centers of frictional resistance. This model has been shown by Zwanzig, Kiefer, and Weiss to possess unphysical singularities when the segmental friction constant is high. Here, it is shown that, for the rigid rod molecule, the singularities are artifacts of the model. The singularities disappear if the segment represented by the point force is small, and if the segment has a finite cross section.
\end{abstract}

The methods of macroscopic hydrodynamics have been applied with considerable success to particles of molecular size. A procedure developed by Kirkwood and Riseman ${ }^{1}$ (KR) from earlier work of Burgers ${ }^{2}$ and Oseen ${ }^{3}$ has been applied widely to polymer solutions, and a variety of results by different workers attest to the success of the procedure. ${ }^{1}$

One of the steps in the KR calculation is based on the solution of a set of simultaneous equations. In an important critical paper, Zwanzig, Kiefer, and Weiss ${ }^{4}$ (ZKW) showed, that for both rigid rod and coiling macromolecules, the equations become singular for certain values of the segmental friction constant. In such a case there is no solution of the equations, and serious doubt is thrown on the logical structure of the Kirkwood-Riseman method.

It is our objective here to show, for the particular case of the rigid rod molecule, that the singularities disappear if the finite cross section of the macromolecule is introduced in the model, and if the segmental unit of the rod is sufficiently small.

In the KR equations for the translational diffusion problem, the mean force exerted on the fluid by segment num- ber $l$ is

$$
\left\langle\mathbf{F}_{l}\right\rangle=-\zeta \mathbf{e}_{x}-\left(\zeta / 6 \pi \eta_{0}\right) \sum_{i=0}^{N} \iota^{\prime}\left\langle 1 / R_{l}\right\rangle\left\langle\mathbf{F}_{s}\right\rangle \quad l=0 \text { to } N
$$

$\zeta$ is the segmental friction constant, $\eta_{0}$ is the viscosity of the solvent, $\mathbf{e}_{x}$ is a unit vector in the $x$ direction, $R_{l s}$ is the distance between segments $l$ and $s$, angular brackets signify an average value taken over all possible orientations and internal configurations of the molecule, $\Sigma^{\prime}$ signifies a sum over all segments $s \neq l$. It is understood that the system is subject to overwhelming Brownian motion, and therefore the molecules are not preferentially oriented. Equation 1 may be written in matrix form

$$
\begin{gathered}
\mathbf{M x}=\mathbf{y} \\
\mathbf{M}=\left\{M_{l s}\right\} \\
M_{l s}=\delta_{l s}+\left(1-\delta_{l s}\right)\left(\zeta / 6 \pi \eta_{0}\right)\left\langle 1 / R_{l s}\right\}
\end{gathered}
$$

A similar set of equations apply to intrinsic viscosity. The vectors $\mathbf{x}$ and $\mathbf{y}$ differ from those of the diffusion problem. However, the point of crucial significance is that the matrix $\mathbf{M}$ remains the same. The existence of a solu- 